\title{
Identification of QTL TGW12 responsible for grain weight in rice based on recombinant inbred line population crossed by wild rice (Oryza minuta) introgression line K1561 and indica rice $\mathrm{G} 1025$
}

Xiaogiong $\mathrm{Li}^{1+}$, Yu Wei ${ }^{1+}$, Jun $\mathrm{Li}^{2+}$, Fangwen Yang ${ }^{3}$, Ying Chen ${ }^{1}$, Yinhua Chen ${ }^{4}$, Sibin Guo ${ }^{1 *}$ and Aihua Sha ${ }^{3^{*}}$ (D)

\begin{abstract}
Background: Limited genetic resource in the cultivated rice may hinder further yield improvement. Some valuable genes that contribute to rice yield may be lost or lacked in the cultivated rice. Identification of the quantitative trait locus (QTL) for yield-related traits such as thousand-grain weight (TGW) from wild rice speices is desired for rice yield improvement.

Results: In this study, sixteen TGW QTL were identified from a recombinant inbred line (RIL) population derived from the cross between the introgression line K1561 of Oryza minuta and the rice cultivar G1025. TGW12, One of most effective QTL was mapped to the region of $204.12 \mathrm{~kb}$ between the marker 2,768,345 and marker 2,853,491 of the specific locus amplified fragment (SLAF). The origin of TGW12 was tested using three markers nearby or within the TGW12 region, but not clarified yet. Our data indicated thirty-two open reading fragments (ORFs) were present in the region. RT-PCR analysis and sequence alignment showed that the coding domain sequences of ORF12, one MADS-box gene, in G1025 and K1561 were different due to alternative slicing, which caused premature transcription termination. The MADS-box gene was considered as a candidate of TGW12.

Conclusion: The effective QTL, TGW12, was mapped to a segment of $204.12 \mathrm{~kb}$ using RlLs population and a MADS-box gene was identified among several candidate genes in the segment. The region of TGW12 should be further narrowed and creation of transgenic lines will reveal the gene function. TGW12 could be applied for improvement of TGW in breeding program.
\end{abstract}

Keywords: Wild rice, Introgression lines, SSR, SLAF, Transcription factor

\section{Background}

Rice (Oryza sativa L.) is the world's most important cereal crop as a staple food [1]. High rice yield is needed to meet the requirement of rapidly increasing population. Grain weight is important to rice yield, and it is usually represented by thousand-grain weight. Many QTLs for rice grain weight have been mapped to rice

\footnotetext{
*Correspondence: sibinguo@163.com; aihuasha@yangtzeu.edu.cn

${ }^{+}$Xiaogiong Li, Yu Wei and Jun Li contributed equally to this work.

${ }^{1}$ Rice Research Institute/Guangxi Key Laboratory of Rice Genetics and

Breeding, Guangxi Academy of Agricultural Science, Nanning 530007,

People's Republic of China

${ }^{3}$ Hubei Collaborative Innovation Center for Grain Industry, Yangtze University, Jingzhou, People's Republic of China

Full list of author information is available at the end of the article
}

chromosomes [2-7]. A dozen of QTLs/genes affecting grain weight have be cloned and functionally charaterized, such as the cytokinin oxidase/dehydrogenase (CKX) OsCKX2(Gn1a) [8], the transmembrane protein GS3 [9, 10 ], and its homolog DEP1 [11], the RING-type E3 ubiquitin ligase GW2 (grain width and weight 2) [12], the arginine-rich domain nuclear protein $q S W 5 / G W 5$ [13], the serine carboxypeptidase GS5/qTGW5a [14], the Kelch-like domain qGL3/ OsPPKL1 [15], the SBP domain transcription factor GW8 (OsSPL16) [16], the IAA (indole-3-acetic acid)- glucose hydrolase protein TGW6 [17], the GNAT-like protein GW6a (Grain weight of chromosome 6)/OsglHAT1 [18], the ABC1-like kinase OsAGSW1 [19], the AP2 transcript factor $q H D 5$ and OsSNB [20, 21], the cytochrome $\mathrm{P} 450$ protein

(c) The Author(s). 2020 Open Access This article is distributed under the terms of the Creative Commons Attribution 4.0 International License (http://creativecommons.org/licenses/by/4.0/), which permits unrestricted use, distribution, and reproduction in any medium, provided you give appropriate credit to the original author(s) and the source, provide a link to the Creative Commons license, and indicate if changes were made. The Creative Commons Public Domain Dedication waiver (http://creativecommons.org/publicdomain/zero/1.0/) applies to the data made available in this article, unless otherwise stated. 
GNS4 [22], the otubain-like protease WTG1 (WIDE AND THICK GRAIN 1) [23], the $16-\mathrm{kDa} \alpha$-amylase/trypsin inhibitor RAG2 [24], the GSK3/SHAGGY-Like Kinase qTGW3 [25]. Some genes such as GW2, WTG1, OsCKX2, TGW3, TGW6, qSW5/GW5qSW5/GW5, GS3, DEP1, qGL3/ $O s P P K L 1$, and $O s S N B$ negatively regulate grain weight, while others like GW6a/OsglHAT1, GNS4, GS5/qTGW5a, OsAGSW1, RAG2, and GW8 (OsSPL16) function as positive regulator of grain weight.

To date, QTLs/genes associated with TGW have been mostly cloned from the cultivated rice. However, it is known that the genetic resource of cultivated rice turned quite limited during the process of wild rice domestication, which may hinder the yield improvement of the cultivated rice. Wild rice species should contain many valuable genes that can be used for genetic improvements of cultivated rice [26]. Thus, the genetic resource of wild rice species should be explored and used for rice high-yield breeding. It would be an effective way to widen the genetic basis of cultivated rice by introduction and application of favorable wild rice genes.

Oryza minuta (O. minuta) is a tetraploid wild rice that possesses a number of favorable yield related genes [27]. Rahman et al. mapped 22 novel yield-related QTLs for 16 agronomic traits using a set of introgression line (IL) of O. minuta, and demonstrated that $57 \%$ of the QTLs were derived from O. minuta [27]. In a previous study, we also detected 28 QTLs for yield-related traits using ILs derived from the backcross of IR24 (O. sativa $L)$ and O. minuta, and found that $46.4 \%$ of notable QTLs were from O. minuta [28].

To further identify the favorable yield-related genes from $O$. minuta, a recombinant inbred line has been developed by crossing of K1561 to indica rice G1025 [28]. K1561 is one out of 192 ILs derived from backcross progenies $\left(\mathrm{BC}_{4} \mathrm{~F}_{2}\right)$ of IR24 and O. minuta. It was produced from one time cross of IR24 and $O$. minuta, then four times backcross with IR24 as recurrent parent, and four times self-cross. K1561 shows excellent agronomic traits such as long panicles and high TGW. G1025 is an excellent restorer line that is widely used in Guangxi Province of China with dense grains but light TGW. In this study, QTL mapping was conducted on the advanced RILs population by SSR and SLAF markers. Thirteen QTLs or TGWs responsible for TGW were detected under fiver environments, and the most effective QTL TGW12 was mapped to the segment of $204.12 \mathrm{~kb}$ based on the high-density genome map constructed with SLAF. The candidate genes of TGW12 were preliminarily concluded, and one gene encoding MADS-box protein was considered as the putative candidate based on sequence alignment. The TGW12 allele for increasing TGW might originated from O. minuta and likely used for rice yield improvement.

\section{Results}

Phenotypic analysis

The two parents G1025 and K1561 showed highly significant differences in TGW under five environments including two locations in Nan-Ning (NN), and Wu-Han $(\mathrm{WH})$, in China with an average of $16.01 \mathrm{~g}$ and $32.07 \mathrm{~g}$ (Table 1), respectively. TGW values of the 201 individuals were mostly distributed between 20 and $30 \mathrm{~g}$ with an average of $24.60 \mathrm{~g}, 25.69 \mathrm{~g}, 22.80 \mathrm{~g}, 24.97 \mathrm{~g}$, and 25.69 $\mathrm{g}$ in 2013NN (2013 in Nan-Ning), 2014NN, 2015NN, 2016NN, 2016WH (in 2016 in Wu-Han)(Table 1, Fig. 1, Additional file 1: Table S1), respectively. TGW values of 104, 106, 104, 107, and 107 out of the 201 individuals were smaller than the average in $2013 \mathrm{NN}, 2014 \mathrm{NN}$, $2015 \mathrm{NN}, 2016 \mathrm{NN}, 2016 \mathrm{WH}$, and TGW of the remaining individuals was either equal to or larger than the average (Additional file 1: Table S1).

\section{QTL mapping of TGW by simple sequence repeats (SSR)}

TGW QTLs were preliminarily detected by 300 SSR markers evenly distributed on the 12 chromosomes. The population were $F_{6}, F_{7}$ RILs derived from the cross of G1025 and K1561 planted in NN in 2013, 2014. Four QTLs TGW3, TGW7, TGW9.2, and TGW12 were stably detected on the chromosomes 3, 7, 9 and 12 in the two environments (Table 2). TGW12 had the greatest effect, which located on the region of RM247 and RM7003 (Table 2), so it was selected for further analysis. There were other 166 SSR markers (Additional file 2: Table S2) in the region based on the genome sequencing data of Nipponbare [29]. The polymorphism of the 166 SSRs was firstly detected between the parental lines G1025 and K1561. As a result, nine SSRs showed polymorphism but only five displayed clear bands. The five SSRs were further used to detect $F_{6}, F_{7}$ RILs population in 2013 and 2014. Finally, TGW12 was mapped to the 5.1 cM region between RM27638 and RM27748 (Fig. 2).

\section{QTL mapping of TGW by SLAF markers}

We have developed 5521 SLAF markers by SLAF sequencing [30]. To further map TGW QTLs, those SLAF markers were used to screen $\mathrm{F}_{8}$ RILs in NN in 2015 and

Table 1 Thousand Grain weight of Parental lines G1025, K1561 and RILS

\begin{tabular}{lllll}
\hline Environments & G1025 $(\mathrm{g})$ & K1561 $(\mathrm{g})$ & $\mathrm{RILS}$ \\
\cline { 4 - 5 } & & & Mean(g) & Range(g) \\
\hline $2013 \mathrm{NN}$ & $16.23 \pm 0.07$ & $31.94 \pm 0.09$ & $24.60 \pm 2.74$ & $16.82-34.67$ \\
$2014 \mathrm{NN}$ & $16.89 \pm 0.09$ & $32.99 \pm 0.12$ & $25.69 \pm 2.90$ & $16.68-36.02$ \\
$2015 \mathrm{NN}$ & $14.44 \pm 0.05$ & $29.58 \pm 0.07$ & $22.80 \pm 2.48$ & $16.67-31.26$ \\
$2016 \mathrm{NN}$ & $15.76 \pm 0.06$ & $33.68 \pm 0.13$ & $24.97 \pm 3.00$ & $15.68-34.05$ \\
$2016 \mathrm{WH}$ & $16.73 \pm 0.09$ & $32.18 \pm 0.11$ & $25.69 \pm 3.04$ & $16.69-35.00$
\end{tabular}

NN Nanning, WH Wuhan 


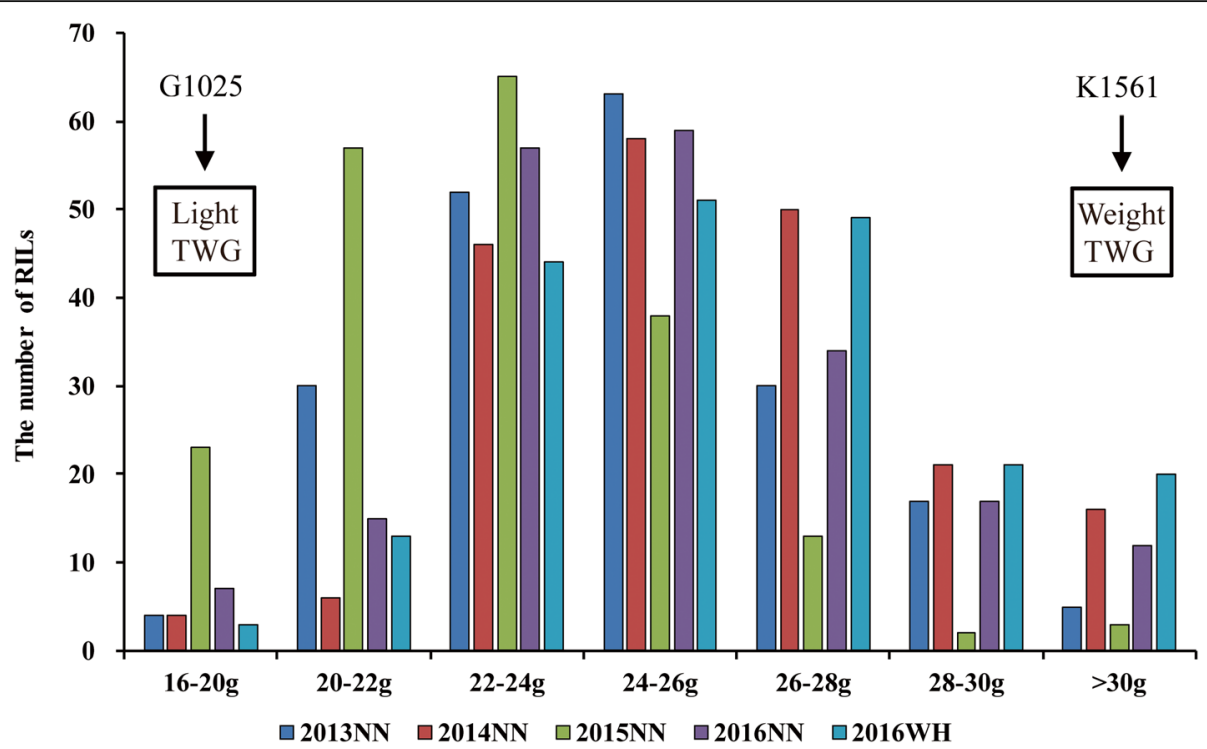

Fig. 1 Phenotypic evaluation of thousand-grain weight (TGW) for G1025, K1561 and RILs. The $Y$ axis represents the number of RIL lines. The $X$ axis represents the values of TGW: $16 \mathrm{~g}<\mathrm{TGW} \leq 20 \mathrm{~g} ; 20<\mathrm{TGW} \leq 22 \mathrm{~g} ; 22 \mathrm{~g}<\mathrm{TGW} \leq 24 \mathrm{~g} ; 24 \mathrm{~g}<\mathrm{TGW} \leq 26 \mathrm{~g} ; 26 \mathrm{~g}<\mathrm{TGW} \leq 28 \mathrm{~g} ; 28 \mathrm{~g}<\mathrm{TGW} \leq$ $30 \mathrm{~g}$; TGW > $30 \mathrm{~g}$. G1025 and K1501 are the parents with light and weight TGW, respectively. The arrows indicate the TGW range of the parents (G1025 and K1561), respectively

$\mathrm{F}_{9}$ RILs in NN and $\mathrm{WH}$ in 2016, respectively. A total of eight QTLs were detected in three environments, namely, TGW7, TGW9.1, and TGW12 in 2015NN; TGW7, TGW9.2, and TGW12 in 2016NN; TGW7 and TGW12 in 2016WH (Table 2, Fig. 3). TGW7 and $T G W 12$ were both detected in three environments, and TGW9.1 and TGW9.2 were each detected once. TGW7 explained the phenotypes for 8.01, 10.76, and 10.43\% inheritance with an LOD of 6.94, 7.69, and 7.48 in $2015 \mathrm{NN}, 2016 \mathrm{NN}$, and $2016 \mathrm{WH}$, respectively, whereas TGW12 showed 22.36, 17.48, and $17.95 \%$ inheritance explaining for the phenotypes with an LOD of 15.42, 11.99 , and 11.96 in the three environments, respectively (Table 2). TGW12 had a greater effect than TGW7, which was consistent with the results analyzed by SSR mapping (mentioned above). Further analysis for

Table 2 Quantitative trait loci (QTL) analysis of rice thousand grain weight

\begin{tabular}{|c|c|c|c|c|c|c|c|c|}
\hline Environments & QTL & Chr & Marker interval & Position (cM) & LOD & Additive effect & $R^{2}(\%)$ & The donor parent \\
\hline \multirow[t]{4}{*}{$2013 N N$} & TGW3 & 3 & RM186-RM416 & 95.7 & - & 0.76 & 5.83 & K1561 \\
\hline & TGW7 & 7 & RM455-RM10 & 76.1 & - & 1.22 & 9.25 & K1561 \\
\hline & TGW9.2 & 9 & RM201-RM6294 & 85.3 & - & 1.05 & 8.07 & K1561 \\
\hline & TGW12 & 12 & RM7003-RM247 & 45.6 & - & 1.93 & 24.81 & K1561 \\
\hline \multirow[t]{4}{*}{ 2014NN } & TGW3 & 3 & RM186-RM416 & 95.7 & - & 0.69 & 4.37 & K1561 \\
\hline & TGW7 & 7 & RM455-RM10 & 76.1 & - & 1.39 & 10.88 & K1561 \\
\hline & TGW9.2 & 9 & RM201-RM6294 & 85.3 & - & 0.85 & 5.34 & K1561 \\
\hline & TGW12 & 12 & RM7003-RM247 & 45.6 & - & 1.71 & 19.12 & K1561 \\
\hline \multirow[t]{3}{*}{ 2015NN } & TGW7 & 7 & Marker1254956 & 99.60 & 6.94 & 0.71 & 8.01 & K1561 \\
\hline & TGW9.1 & 9 & Marker864241 & 166.79 & 9.15 & 0.84 & 11.21 & K1561 \\
\hline & TGW12 & 12 & Marker2768345 & 56.06 & 15.42 & 1.18 & 22.36 & K1561 \\
\hline \multirow[t]{3}{*}{ 2016NN } & TGW7 & 7 & Marker1254956 & 99.60 & 7.69 & 0.99 & 10.76 & K1561 \\
\hline & TGW9.2 & 9 & Marker775977 & 163.15 & 8.91 & 1.09 & 12.95 & K1561 \\
\hline & TGW12 & 12 & Marker2768345 & 56.06 & 11.99 & 1.26 & 17.48 & K1561 \\
\hline \multirow[t]{2}{*}{ 2016WH } & TGW7 & 7 & Marker1124977 & 101.91 & 7.48 & 0.99 & 10.43 & K1561 \\
\hline & TGW12 & 12 & Marker2768345 & 56.06 & 11.96 & 1.30 & 17.95 & K1561 \\
\hline
\end{tabular}




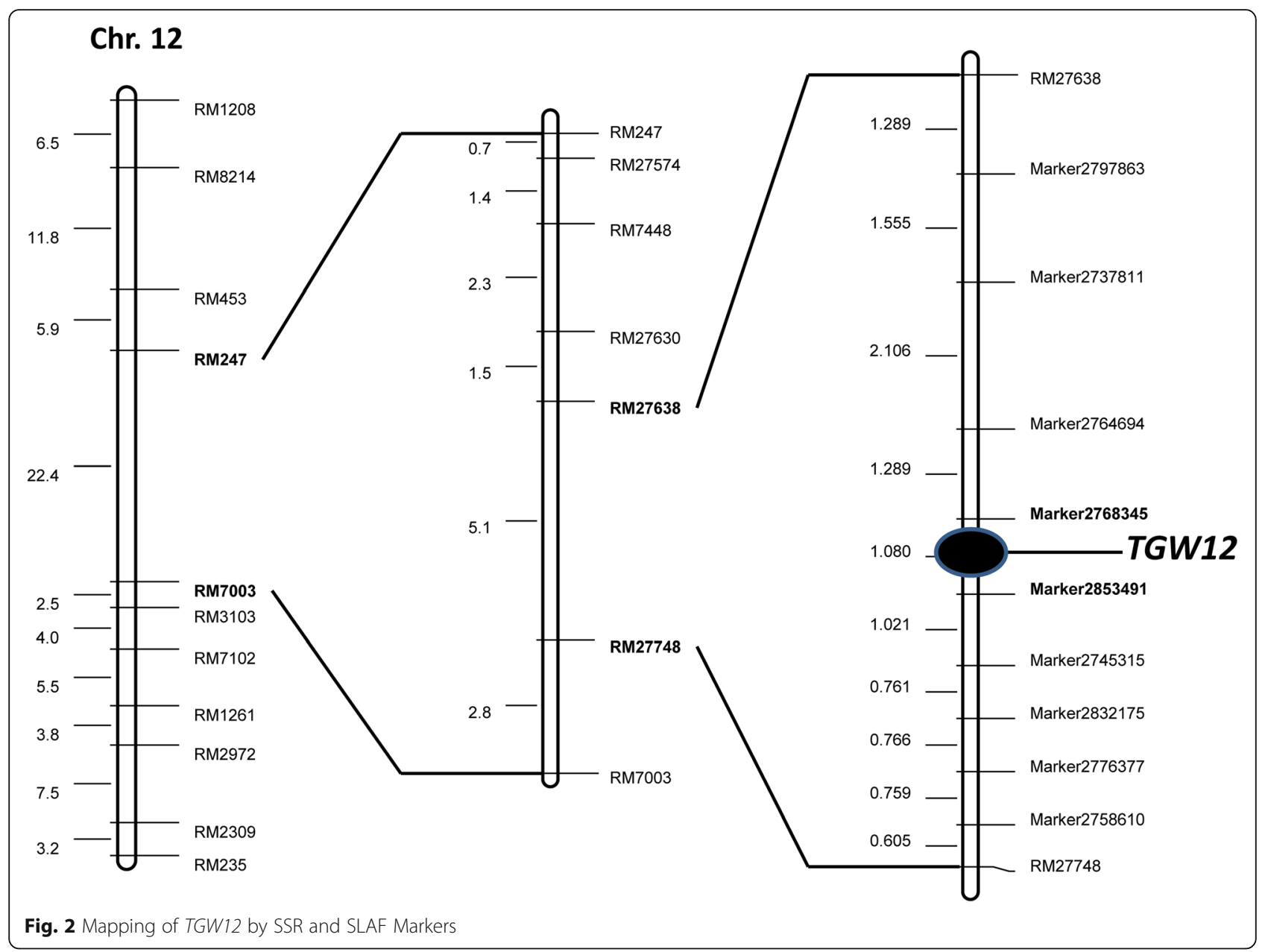

TGW12 was conducted by comparing the linkage map constructed by SSR and SLAF markers. Consequentially, nine SLAF markers fell into the region of RM27638RM27748, and TGW12 was further narrowed to 204.12 $\mathrm{kb}$ region between SLAFs Marker 2,768,345 and Marker 2,853,491 (Fig. 2).

\section{Evaluation of TGW12 phenotype and genetic origin identification of TGW12 segment}

In order to evaluate whether the phenotypes were determined by TGW12, 16 out of the 201 RILs containing the $T G W 12$ region were identified by means of the markers nearby the region (Fig. 4). Then, the phenotypes and genotypes of the 16 RILs were compared. All the 16 RILs with one or two segments of K1561 showed TGW increase than the recurrent parent G1025, suggesting TGW12 control TGW (Fig. 4). To clarify whether the increasing effect of TGW12 was originated from O. minuta, the genotypes of G1025, K1561, IR24, and O. minuta were examined using markers nearby or within TGW12. The genotype of K1561 was the same as that of IR24 but different from that of G1025 and O. minuta on the sites of
RM27638 and RM27748, which are nearby TGW12 (Figs. 2 and 5). However, the genotype of K1561 was the same as those of IR24 and O. minuta, but it was different from that of G1025 at the site of Marker 2,768,345, which is linked with $T G W 12$. It was hard to draw a conclusion whether TGW12 originated from IR24 or O. minuta based on the above results. It has been suggested that translocation through centric break-fusion occurred more frequently than recombination in the introgression lines with interspecific cross, which didn't always result in an $O$. minuta chromosome arm onto a complete or incomplete O. sativa chromosome [30, 31]. Thus, TGW12 origination remains to be determined in the near future. It is feasible to compare sequence of $T G W 12$ candidate among $O$. minuta, IR24, and K1561 once it was fine mapped.

\section{Preliminary prediction of candidate genes for TGW12}

Analysis of annotated genes indicated that 32 ORFs located in the $204.12 \mathrm{~kb}$ region based on Nipponbare genome annotation (http://rice.plantbiology.msu.edu) (Table 3). Among them, 13 ORFs encoded functional proteins and 19 ORFs were annotated as transposon/retrotransposon proteins, 

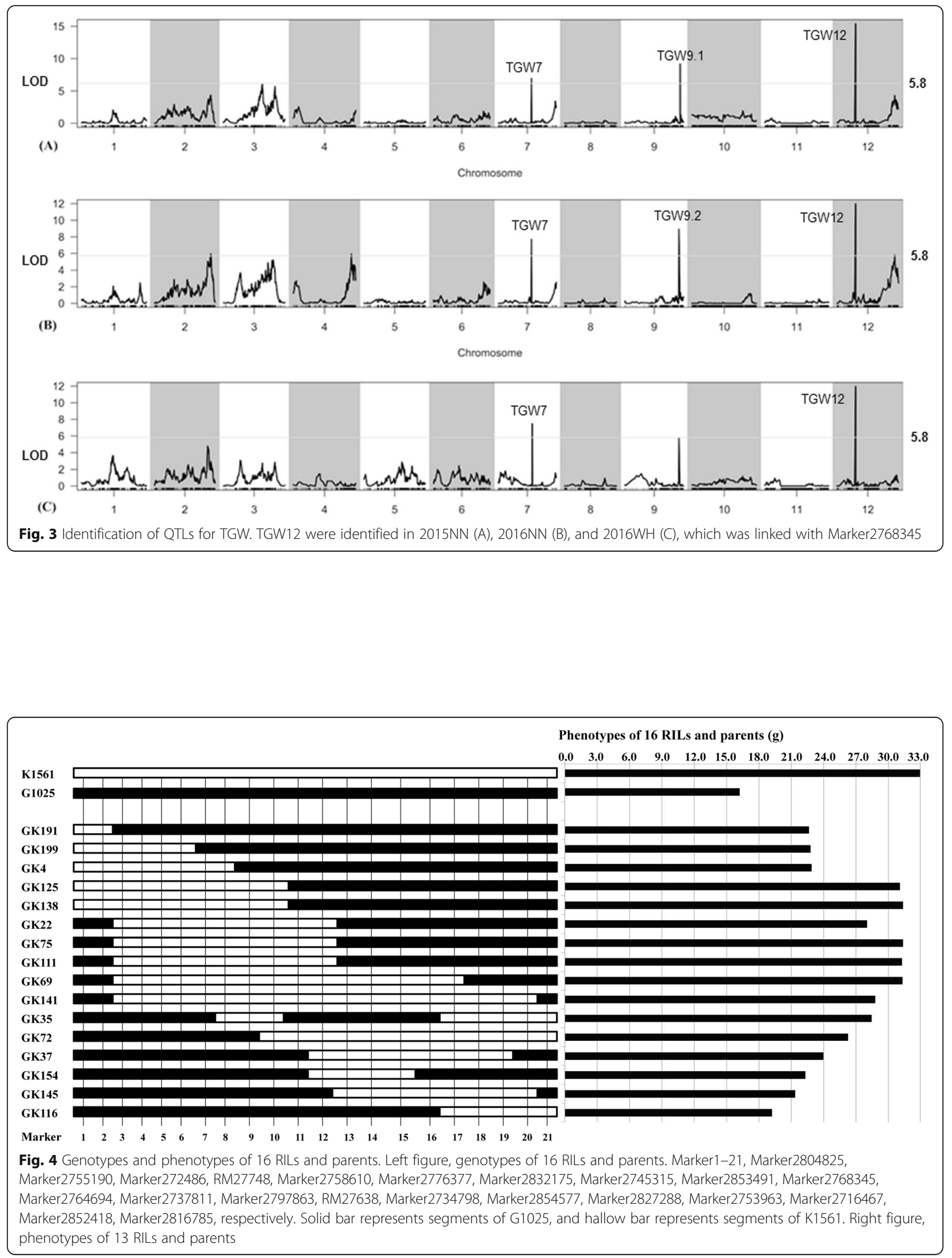


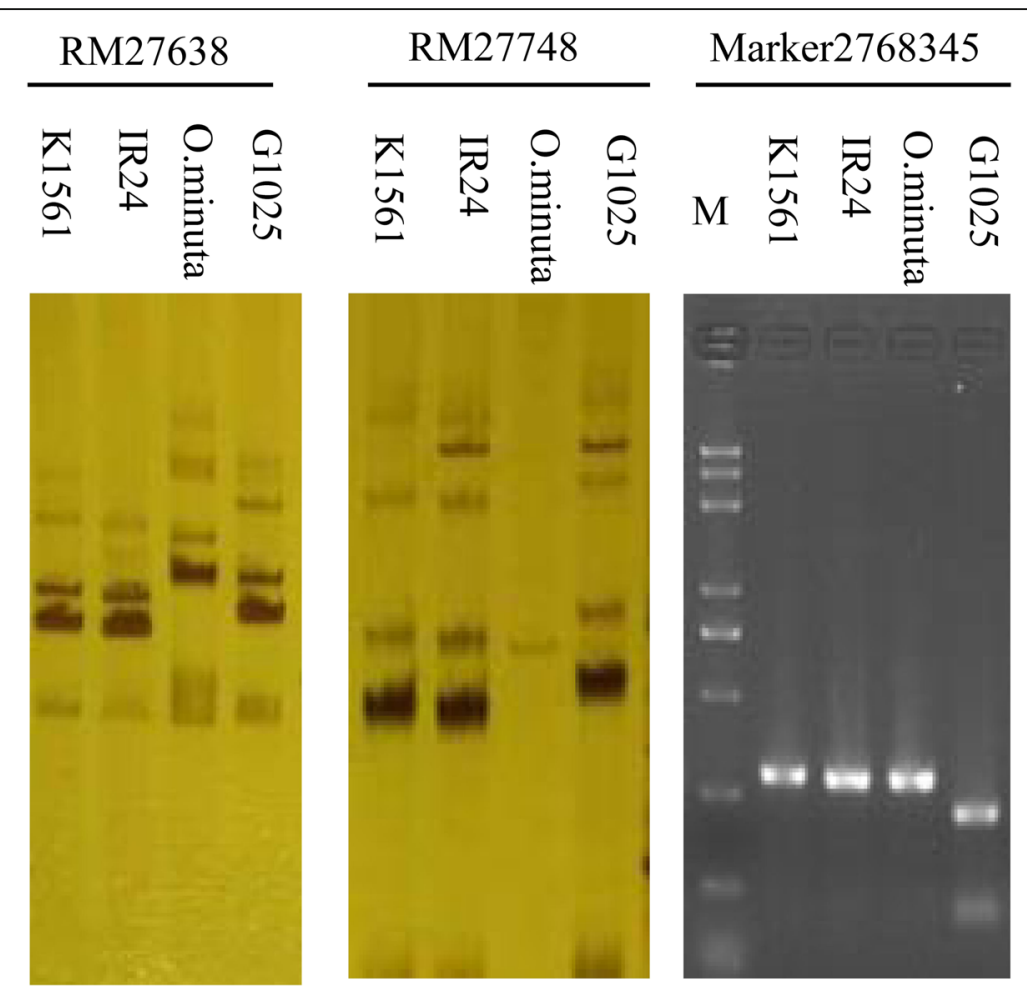

Fig. 5 Identification of TGW12 segment by SSR or SLAF markers. Lanes1-4, K1561, IR24, O. minuta, G1025. M, DNA marker 2000 plus

hypothetical proteins, or expressed protein. It is worth noting that there were four transcription factors (TFs) among the functional proteins: two MADS-box proteins (ORF12, ORF14), one ZF-HD protein (ORF24), and one B-box zinc finger protein (ORF27). Because TFs play crucial roles in regulation of plant growth and development [32], the four TFs were considered preferentially as putative candidate genes of TGW12. Reverse transcript (RT)-PCR were conducted to amplify the CDS (coding domain sequence) of the four transcription factors from the parents G1025 and

Table 3 Predicted candidate genes of TGW12

\begin{tabular}{llll}
\hline ORF5 & Gene product & ORFs & Gene product \\
\hline ORF1 & NB-ARC/LRR disease resistance protein & ORF17 & ATP synthase subunit beta \\
ORF2 & Ty3-gypsy retrotransposon protein & ORF18 & Ribulose bisphosphate carboxylase large chain precursor \\
ORF3 & Ty3-gypsy retrotransposon protein & ORF19 & OsClp13 - Putative Clp protease homologue \\
ORF4 & Ty3-gypsy retrotransposon protein & ORF20 & Photosystem II P680 chlorophyll A apoprotein \\
ORF5 & expressed protein & ORF21 & Retrotransposon protein \\
ORF6 & rp1 & ORF22 & Ty3-gypsy retrotransposon protein \\
ORF7 & expressed protein & ORF23 & Ty3-gypsy retrotransposon protein \\
ORF8 & hypothetical protein & ORF24 & ZF-HD protein \\
ORF9 & expressed protein & ORF25 & uncharacterized protein ycf45 \\
ORF10 & Ty1-copia retrotransposon protein & ORF26 & expressed protein \\
ORF11 & expressed protein & ORF27 & B-box zinc finger family protein \\
ORF12 & MADS-box family gene & ORF28 & AAA-type ATPase family protein \\
ORF13 & expressed protein & ORF29 & Retrotransposon protein \\
ORF14 & MADS-box family gene & ORF30 & expressed protein \\
ORF15 & CACTA transposon protein & ORF31 & expressed protein \\
ORF16 & Clathrin adaptor complex small chain domain containing protein & ORF32 & NB-ARC domain containing protein \\
\hline
\end{tabular}


K1561. Sequence comparison indicated that the amplified sequence of ORF12 in K1561 was 56 bp shorter than that of G1025, which resulted in premature transcription termination, consequently leading to a peptide with 45 amino acid residues only in K1561, whereas ORF12 in G1025 encoded a protein with 202 amino acid residues (Additional file 3: Figure S1). Further analysis revealed that lack of $56 \mathrm{bp}$ of ORF12 in K1561 was due to alternative splicing (AS) in the first extron (Fig. 6b, c), which causes premature termination of ORF12 translation (Additional file 3: Figure S1). There were no difference in the CDS of ORF14, ORF24, and ORF27 between K1561 and G1025 (data not shown). Thus, the MADS-box (ORF12) was likely one putative candidate of $T G W 12$. However, possibility of other nine functional proteins and the hypothetical proteins, or expressed protein independently or collectively affecting TGW could not be excluded. Further investigation is required.

\section{Discussion}

Rice is one of the most important staple food widely consumed by one-half world's peopulation and more product is needed as population increases in future. However, further yield improvement of rice is constrained by the narrow genetic basis of cultivated rice varieties. Wild rice species are good candidates to explore the genetic resource for valuably genes to further enhance rice productivity [26].
O. minuta possesses a number of outstanding genes associated with resistance and yield [27]. Lots of QTLs for yield related traits have been identified using the introgression lines (IL) consisting of $O$. minuta segments $[27,28]$. In this study, sixteen TGWs were detected using the advanced RILs population under five environments (Table 2). Among them, the most effective QTL, TGW12, was mapped on chromosome 12. Eight QTLs for TGW (AQAG040, AQCF014, AQAG053, AQGP079, AQDR045, AQDR047, AQCY020, CQAS153) had been previously mapped to Chromosome 12 (http://archive. gramene.org/). Location comparison indicated that TGW12 was partially overlapped with AQDR045. TGW12 and AQDR045 were respectively located in the regions of 4,037,811-6,150,143 and 1,589,200-5, 829,185 on Chromosome 12 in the physical map of Nipponbare genome [29], suggesting that there might be a major QTL controlling grain weigh in this region. However, AQDR045 was mapped by using the population derived from two cultivated rice Lemont and Teqing [33], whereas TGW12 was mapped using the population of one O. minuta introgression line and one cultivated line. Although it was uncertain whether TGW12 allele originated from O. minuta or IR24, it showed a great increasing effect for TGW and could be directly applied in the breeding program.

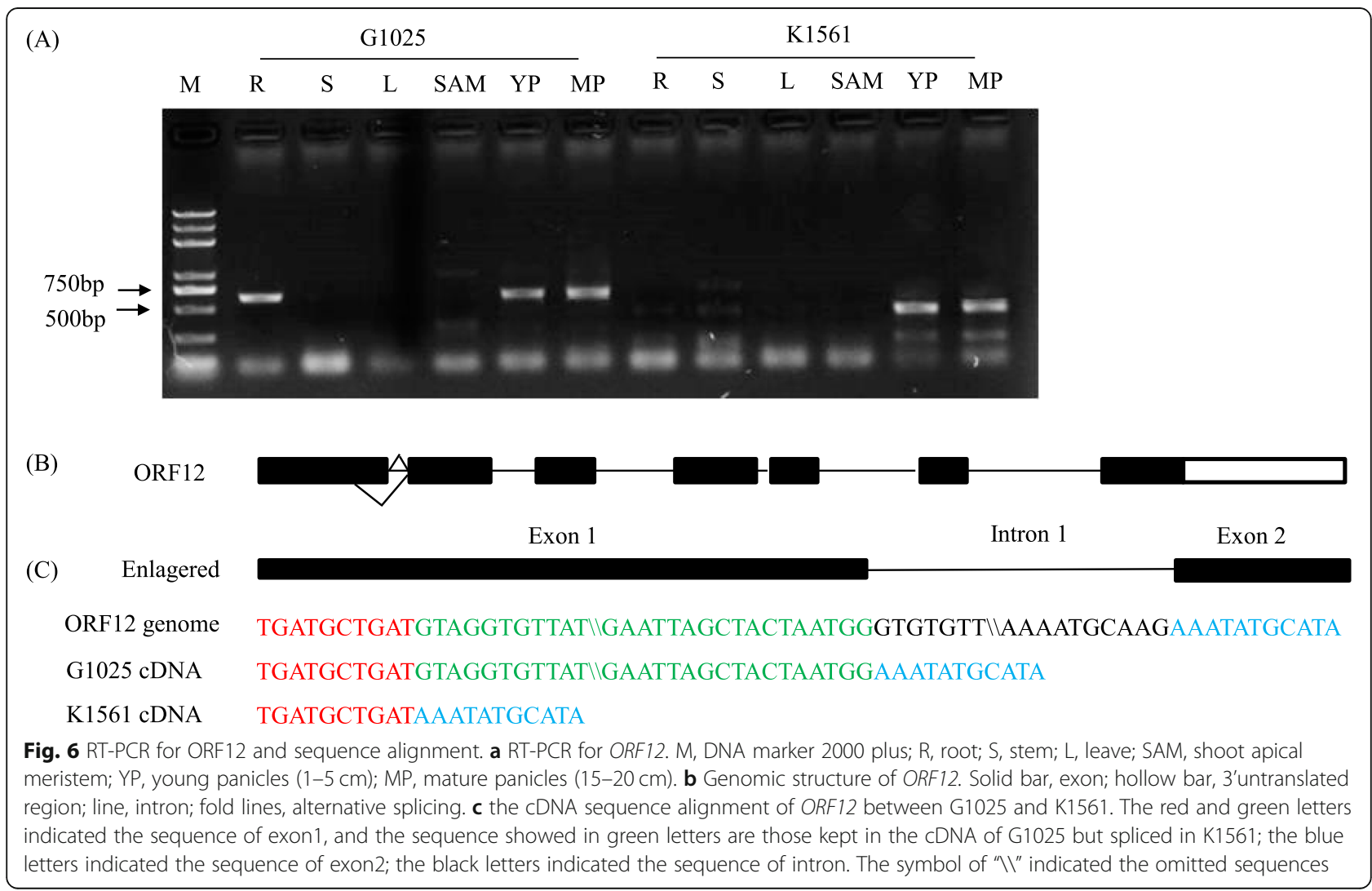


There were 32 annotated ORFs in the TGW12 region, among which four ORFs were identified as TFs based on sequence alignment and considered as TGW12 candidates due to their regulatory roles in plant growth and development. Sequence analysis indicated that ORF12 in K1561 was truncated, with 45 amino acid residues only, due to an AS event while the counterpart in G1025 possessed a full length of the protein. No sequence difference in the other three TFs (ORF14, ORF24, and ORF27) was found between K1561 and G1025. ORF12 encoded a MADS-box protein, which possesses a highly conserved DNA-binding MADS domain and is involved predominantly in developmental processes [34]. In Arabidopsis, there are 107 genes encoding MADS-box proteins [34], and almost all of them are involved in the process of flower and seed development [35]. In rice, 75 MADS-box genes were identified, and more than 20 were transcribed during the stages of panicle and seed development [36]. In addition, alternative splicing of one MADS-box transcription factor OsMADS1 encoded by OsLG3b (Os03g0215400) controls grain length and yield in japonica rice [37]. Our results suggested that ORF12 could be critical for the function of TGW12, even functioned as $T G W 12$. However, further studies are required to examine the function of other ORFs located in the region.

\section{Conclusions}

In this study, an effective QTL TGW12 related to the trait of thousand-grain weight in rice was mapped to a segment with $204.12 \mathrm{~kb}$ using RILs population derived from the cross progenies of one $O$. minuta introgression line and one cultivated rice. Out of 32 ORFs located in the region of TGW12, ORF12 encoded a MADS-box protein could be crucial for the TGW12 function. Further investigation is required to validate this speculation.

\section{Methods}

\section{Plant materials and field trials}

O. minuta (Acc. No. 101133) and IR24 were kindly provided by the International Rice Germplasm Centre of the International Rice Research Institute. The parental line G1025 was kindly provide by Rice Research Institute of Guangxi Academy of Agricultural Science. The parental lines K1561 was developed by our lab in Rice Research Institute of Guangxi Academy of Agricultural Science. It is produced by one time cross of IR24 and $O$. minuta, then four times backcross with IR24 as recurrent parent, and four times self-cross. The parental lines G1025 and K1561 along with $201 \mathrm{~F}_{6}, \mathrm{~F}_{7}$ RILs were planted in Nanning (NN) from February to July in 2013 and 2014, respectively. The parental lines along with 201 $\mathrm{F}_{8}$ RILs were planted in NN (February to July) in 2015, and parents and $\mathrm{F}_{9}$ RILs were planted in NN (February to July) and Wuhan (WH) from May to October in
2016, respectively. The phenotypes of parents and RILs were collected to map TGW based on SSR or SLAFs. Grain weight was calculated based on 200 grains and converted to TGW after harvesting and sun-drying. The mean values of ten plants were used as input data to identify QTLs (Additional file 1: Table S1).

\section{SSR, linkage, and QTL analysis}

DNA was extracted from fresh leaves following the CTAB procedure [38]. SSR markers were used to analyze a polymorphism between the parents (Additional file 2: Table S2). SSR were synthesized according to published sequences [29]. Polymerase chain reaction (PCR) was conducted in a $15 \mu \mathrm{L}$ volume as follow: $50 \mathrm{ng}$ of template DNA, $0.3 \mu \mathrm{L}$ of $10 \mathrm{mM}$ each dNTPs, 0.5 units of Taq DNA polymerase, $1.5 \mu \mathrm{L}$ of $10 \times$ PCR buffer with $\mathrm{Mg}^{2+}$, and $0.5 \mu \mathrm{L}$ of $10 \mu \mathrm{M}$ forward and reverse primers. The reaction conditions was carried out as an initial denaturation at $94{ }^{\circ} \mathrm{C}$ for $5 \mathrm{~min}$, followed by 35 cycles of $30 \mathrm{~s}$ at $94^{\circ} \mathrm{C}, 30 \mathrm{~s}$ at $56^{\circ} \mathrm{C}$, and $30 \mathrm{~s}$ at $72^{\circ} \mathrm{C}$, with a final extension at $72^{\circ} \mathrm{C}$ for $10 \mathrm{~min}$. PCR products were separated on $6 \%$ polyacrylamide denaturing gels, and the bands were revealed by the silver-staining protocol [39].

Linkage was constructed by Mapmaker/Exp 3.0 [40]. Genetic distance was calculated by the Kosambi function. QTLNetwork2.2 was used to analyze QTL at a threshold of LOD 3.0 [41].

\section{Single nucleotide polymorphism (SNP) genotyping, linkage map construction and QTL analysis}

Genomic DNA was extracted from fresh leaves of the parents and RILs by CTAB [38]. Quantified DNA was used for SLAF sequencing by an Illumina HiseqTM 2500 [42]. SLAF markers, developed in previous work, were used for genotyping, linkage map construction and QTL analysis for TGW in this study as described by Zhu et al. [30].

\section{Derived cleaved amplified polymorphic sequences (dCAPS) marker development}

dCAPS marker was developed for SLAF Marker2768345. Primers were designed according to dCAPS Finder 2.0 (http://helix.wustl.edu/dcaps/dcaps.html). PCR were conducted in a $20 \mu \mathrm{L}$ volume as follow: $100 \mathrm{ng}$ of template DNA, $0.5 \mu \mathrm{L}$ of $10 \mathrm{mM}$ each dNTPs, 1 units of Taq DNA polymerase, $2.0 \mu \mathrm{L}$ of $10 \times$ PCR buffer with $\mathrm{Mg}^{2+}$, and $0.5 \mu \mathrm{L}$ of $10 \mu \mathrm{M}$ forward and reverse primers. The reaction conditions was carried out as an initial denaturation at $94{ }^{\circ} \mathrm{C}$ for $3 \mathrm{~min}$, followed by 35 cycles of $30 \mathrm{~s}$ at $94{ }^{\circ} \mathrm{C}, 30 \mathrm{~s}$ at $60{ }^{\circ} \mathrm{C}$, and $30 \mathrm{~s}$ at $72^{\circ} \mathrm{C}$, with a final extension at $72{ }^{\circ} \mathrm{C}$ for $5 \mathrm{~min}$. PCR products were digested with EcoR V (Takara, China) for $4 \mathrm{~h}$ at $37^{\circ} \mathrm{C}$, then were resolved in $2 \%$ agrose gel to genotype. 


\section{Candidate gene prediction and RT-PCR analysis}

The predicted genes in the target region of QTL were analyzed according to the annotation of Nipponbare reference genome2. RT-PCR was conducted as described by Sha et al. [43]. In brief, total RNA was extracted from roots, leaves, stems, shoot apical meristem, young panicles $(1-5 \mathrm{~cm})$, mature panicles $(15-20 \mathrm{~cm})$ of $\mathrm{K} 1561$ and G1025 with Trizol (Invitrogen, Carlsbad, CA, USA). RNAs were digested with DNase I (Promega, USA) to eliminate genomic DNA contamination before cDNA synthesis. For cDNA synthesis, $5.0 \mu \mathrm{g}$ total RNA was used for reverse transcribed by M-MLV Reverse Transcriptase (Promega, USA) using oligo-d (T) according to the user manual. For RT-PCR analysis, $2 \mu \mathrm{l}$ of the first cDNA strand was used with gene-specific primer pairs in $20 \mu \mathrm{L}$ reaction volumes, which contained $1 \mu \mathrm{M}$ forward and reverse primer each, $200 \mu \mathrm{M}$ dNTPs, and $1 \mathrm{U}$ Taq enzyme (Takara, China). A DNA Engine peltier ThermalCycler (Bio-RAD) was used to perform the reaction. The PCR procedure was as follows: $94^{\circ} \mathrm{C}$ for $2 \mathrm{~min}$; 35 cycles of $94{ }^{\circ} \mathrm{C}$ for $30 \mathrm{~s}, 53^{\circ} \mathrm{C}$ for $30 \mathrm{~s}, 72^{\circ} \mathrm{C}$ for $1 \mathrm{~min}$ per $1 \mathrm{~kb} ; 5 \mathrm{~min}$ for a final elongation at $72^{\circ} \mathrm{C}$. The PCR products were purified from agarose gel after electrophoresis and cloned into pMD-18 T vector (Takara, China). Clones were sequenced by Tianyihuiyuan Company (Wuhan, China). Sequences were aligned by ClustalW (https://www.genome.jp/tools-bin/clustalw).

\section{Supplementary information}

Supplementary information accompanies this paper at https://doi.org/10 1186/s12863-020-0817-x.

Additional file 1: Table S1. Phenotypes of parents and RILs in different environments.

Additional file 2: Table S2. SSR primer sequences used in the present study.

Additional file 3: Figure S1. Alignment of amino acid sequences of ORF12 between G1025 and K1561.

\begin{abstract}
Abbreviations
CKX: Cytokinin oxidase/dehydrogenase; dCAPS: Derived cleaved amplified polymorphic sequences; GW2: Grain width and weight 2; GW6a: Grain weight of chromosome 6; IAA: Indole-3-acetic acid; ORFs: Open reading fragments; PCR: Polymerase chain reaction; QTL: Quantitative trait locus; RIL: Recombinant inbred line; RT-PCR: Reverse transcript (RT)-PCR; SLAF: Specific locus amplified fragment; SNP: Single nucleotide polymorphism; SSR: Simple sequence repeats; TGW: Thousand-grain weight;
\end{abstract} WTG1: Wide and thick grain 1

\section{Acknowledgements}

Not applicable.

\section{Authors' contributions}

$\mathrm{XL}$ and $\mathrm{YW}$ conducted SLAF analysis. FY performed SSR analysis. YC and JL conducted phenotype analysis. YHC predicted the candidate genes. AS and SG designed the experiments. $\mathrm{XL}, \mathrm{YW}$, and FY wrote the manuscript. All authors read and approved the final manuscript.

\section{Funding}

This work was supported by grants from the National Science and Technology Major Project of China (2016ZX08001001), National Natural Science Fund of China (No. 31860368, 31771735, 31960402), Guangxi Natural Science Foundation of China (No. 2017GXNSFDA198039), and Guangxi Key Laboratory of Rice Genetics and Breeding Open Foundation of China (No. 160-380-16-1). The funders had no role in the study design, data collection, and analysis, decision to publish or preparation of the manuscript.

\section{Availability of data and materials}

The datasets used and/or analyzed during the current study are available from the corresponding author on reasonable request.

\section{Ethics approval and consent to participate}

The research was conducted in accordance with the IUCN Policy Statement on Research Involving Species at Risk of Extinction and the Convention on the Trade in Endangered Species of Wild Fauna and Flora.

\section{Consent for publication}

Not applicable.

Competing interests

The authors declare that they have no competing interests.

\section{Author details}

${ }^{1}$ Rice Research Institute/Guangxi Key Laboratory of Rice Genetics and Breeding, Guangxi Academy of Agricultural Science, Nanning 530007, People's Republic of China. ${ }^{2}$ Oil Crops Research Institute of Chinese Academy of Agricultural Science, Wuhan 430062, People's Republic of China. ${ }^{3}$ Hubei Collaborative Innovation Center for Grain Industry, Yangtze University, Jingzhou, People's Republic of China. ${ }^{4}$ Hainan Key Laboratory for Sustainable Utilization of Tropical Bioresource, Hainan University, Haikou 570228, People's Republic of China.

Received: 5 June 2019 Accepted: 29 January 2020

Published online: 03 February 2020

\section{References}

1. Khush G. Green revolution: the way forward. Nat Rev Genet. 2001;2:815-22.

2. Guo L, Ma L, Jiang H, Zeng D, Hu J, Wu L, et al. Genetic analysis and fine mapping of two genes for grain shape and weight in rice. J Integr Plant Biol. 2009;51:45-51.

3. Liu T, Shao D, Kovi M, Xing Y. Mapping and validation of quantitative trait loci for spikelets per panicle and 1,000-grain weight in rice (Oryza sativa L.). Theor Appl Genet. 2010;120:933-42.

4. Bai X, Luo L, Yan W, Kovi M, Xing Y. Quantitative trait loci for rice yieldrelated traits using recombinant inbred lines derived from two diverse cultivars. J Genet. 2011;90:209-15.

5. Tang S, Shao G, Wei X, Chen M, Sheng Z, Luo J, et al. QTL mapping of grain weight in rice and the validation of the QTL qTGW3.2. Gene. 2013;27:201-6.

6. Xu F, Sun $X$, Chen $Y$, Huang $Y$, Tong C, Bao J. Rapid identification of major QTLS associated with rice grain weight and their utilization. PLoS One. 2015; 10(3):e0122206.

7. Zhang H, Fan Y, Zhu Y, Chen J, Yu S, Zhuang J. Dissection of the gTGW1.1 region into two tightly-linked minor QTLs having stable effects for grain weight in rice. BMC Genet. 2016;17:98.

8. Ashikari M, Sakakibara H, Lin S, Yamamoto T, Takashi T, Nishimura A, et al. Cytokinin oxidase regulates rice grain production. Science. 2005:309:741-5.

9. Fan C, Xing Y, Mao H, Lu T, Han B, Xu C, et al. GS3, a major QTL for grain length and weight and minor QTL for grain width and thickness in rice, encodes a putative transmembrane protein. Theor Appl Genet. 2006;112: $1164-71$.

10. Mao H, Sun S, Yao J, Wang C, Yu S, Xu C, et al. Linking differential domain functions of the GS3 protein to natural variation of grain size in rice. Proc Natl Acad Sci U S A. 2010;107(45):19579-84.

11. Huang X, Qian Q, Liu Z, Sun H, He S, Luo D, et al. Natural variation at the DEP1 locus enhances grain yield in rice. Nat Genet. 2009:41:494-7.

12. Song $X$, Huang W, Shi M, Zhu M, Lin H. A QTL for rice grain width and weight encodes a previously unknown RING-type E3 ubiquitin ligase. Nat Genet. 2007;39:623-30 
13. Shomura A, Izawa T, Ebana K, Ebitani T, Kanegae H, Konishi S, et al. Deletion in a gene associated with grain size increased yields during rice domestication. Nat Genet. 2008:40:1023-8.

14. Li Y, Fan C, Xing Y, Jiang Y, Luo L, Sun L, et al. Natural variation in GS5 plays an important role in regulating grain size and yield in rice. Nat Genet. 2011; 43:1266-9.

15. Zhang X, Wang J, Huang J, Lan H, Wang C, Yin C, et al. Rare allele of OSPPKL1 associated with grain length causes extra-large grain and a significant yield increase in rice. Proc Natl Acad Sci U S A. 2012;109(52): 21534-9.

16. Wang S, Wu K, Yuan Q, Liu X, Liu Z, Lin X, et al. Control of grain size, shape and quality by OSSPL16 in rice. Nat Genet. 2012;44:950-4.

17. Ishimaru K, Hirotsu N, Madoka Y, Murakami N, Hara N, Onodera H, et al. Loss of function of the IAA-glucose hydrolase gene TGW6 enhances rice grain weight and increases yield. Nat Genet. 2013;45:707-11.

18. Song X, Kuroha T, Ayano M, Furuta T, Nagai K, Komeda N, et al. Rare allele of a previously unidentified histone $\mathrm{H} 4$ acetyltransferase enhances grain weight, yield, and plant biomass in rice. Proc Natl Acad Sci U S A. 2015;112:76-81.

19. Li T, Jiang J, Zhang S, Shu H, Wang Y, Lai J, et al. OsAGSW1, an ABC1-like kinase gene, is involved in the regulation of grain size and weight in rice. J Exp Bot. 2015;66:5691-701.

20. Sun B, Zhan X, Lin Z, Wu W, Yu P, Zhang Y, et al. Fine mapping and candidate gene analysis of QHD5, a novel major QTL with pleiotropism for yield-related traits in rice (Oryza sativa L.). Theor Appl Genet. 2017;130:247-58.

21. Ma X, Feng F, Zhang $Y$, Elesawi IE, Xu K, Li T, et al. A novel rice grain size gene OsSNB was identified by genome-wide association study in natural population. PLoS Genet. 2019;15(5):e1008191.

22. Zhou Y, Tao Y, Zhu J, Miao J, Liu J, Liu Y, et al. GNS4, a novel allele of DWARF11, regulates grain number and grain size in a high-yield rice variety. Rice. 2017:10:34.

23. Huang K, Wang D, Duan P, Zhang B, Xu R, Li N, et al. WIDE AND THICK GRAIN 1, which encodes an otubain-like protease with deubiquitination activity, influences grain size and shape in rice. Plant J. 2017;91:849-60.

24. Zhou W, Wang X, Zhou D, Ouyang Y, Yao J. Overexpression of the 16-kDa a-amylase/trypsin inhibitor RAG2 improves grain yield and quality of rice. Plant Biotechnol J. 2017;15:568-80.

25. Hu Z, Lu S, Wang M, He H, Sun L, Wang H, et al. A novel QTL qTGW3 encodes the GSK3/SHAGGY-like kinase OsGSK5/OsSK41 that interacts with OsARF4 to negatively regulate grain size and weight in rice. Mol Plant. 2018; 11:736-49.

26. Guo S, Qin F, Zhang D, Lin X. Characterization of interspecific hybrids and backcross progenies from a cross between Oryza minuta and Oryza sativa. Sci China Ser C-Life Sci. 2009;52:1148-55.

27. Rahman M, Chu S, Choi M, Qiao Y, Jiang W, Piao R, et al. Identification of QTLs for some agronomic traits in rice using an introgression line from Oryza minuta. Mol Cells. 2007;24:16-26.

28. Guo S. Development and characterization of substation lines from a cross of Oryza sativa and Oryza minuta. Wuhan: Dissertation thesis, Huazhong Agricultural University; 2009.

29. International Rice Genome Sequencing Project. The map-based sequence of the rice genome. Nature. 2005;436:793-800.

30. Zhu Z, Li X, WeiY GS, Sha A. Identification of a novel QTL for panicle length from wild rice (Oryza minuta) by Specific Locus Amplified Fragment Sequencing and high density genetic mapping. Front Plant Sci. 2018;9:1492.

31. Romero G, Amante-Bordeos A, Dalmacio R, Elloran R, Sitch L. Comparative studies of isozymes in Oryza sativa, O. minuta, and their interspecific derivatives: evidence for homoeology and recombination. Theor Appl Genet. 1993:87:609-15

32. Sasaki K. Utilization of transcription factors for controlling floral morphogenesis in horticultural plants. Breed Sci. 2018;68:88-98.

33. Li Z, Pinson S, Park W, Paterson A, Stansel J. Epistasis for three grain yield components in rice (Oryza sativa L.). Genetics. 1997;145:453-65.

34. Parenicova L, de Folter S, Kieffer M, Horner D, Favalli C, Busscher J, et al. Molecular and phylogenetic analyses of the complete MADS-box transcription factor family in Arabidopsis: new openings to the MADS world. Plant Cell. 2003;15:1538-51.

35. de Folter S, Busscher J, Colombo L, Losa A, Angenent G. Transcript profiling of transcription factor genes during silique development in Arabidopsis. Plant Mol Biol. 2004;56:351-66.

36. Arora R, Agarwal P, Ray S, Kumar Singh A, Singh V, Tyagi A, et al. MADS-box gene family in rice: genome-wide identification, organization and expression profiling during reproductive development and stress. BMC Genomics. 2007:8:242

37. Yu J, Miao J, Zhang Z, Xiong H, Zhu X, Sun X, et al. Alternative splicing of OsLG3b controls grain length and yield in japonica rice. Plant Biotechnol J. 2018;16(9):1667-78.

38. Murray $\mathrm{M}$, Thompson W. Rapid isolation of high molecular-weight plant DNA. Nucleic Acids Res. 1980:8:4321-5.

39. Cho Y, Blair M, Panaud O, McCouch S. Cloning and mapping of varietyspecific rice genomic DNA sequences: amplified fragment length polymorphisms (AFLP) from silver-stained polyacrylamide gels. Genome. 1996;39:373-8.

40. Lincoln S, Daly M, Lander E. Constructing a genetic linkage map with MAPMAKER/EXP v. 3.0: a tutorial and reference manual. Whitehead Institute Technical Report, Cambridge, Mass; 1993.

41. Yang J, Zhu J. Methods for predicting superior genotypes under multiple environments based on QTL effects. Theor Appl Genet. 2005;110(7):1268-74

42. Sun X, Liu D, Zhang X, Li W, Liu H, Hong W, et al. SLAF-seq: an efficient method of large-scale de novo SNP discovery and genotyping using highthroughput sequencing. PLoS One. 2013:8:e58700.

43. Sha A, Qi Y, Shan Z, Chen H, Yang Z, Qiu D, et al. Identifying patellin-like genes in Glycine max and elucidating their response to phosphorus starvation. Acta Physiol Plant. 2016:38:138.

\section{Publisher's Note}

Springer Nature remains neutral with regard to jurisdictional claims in published maps and institutional affiliations.

\section{Ready to submit your research? Choose BMC and benefit from:}

- fast, convenient online submission

- thorough peer review by experienced researchers in your field

- rapid publication on acceptance

- support for research data, including large and complex data types

- gold Open Access which fosters wider collaboration and increased citations

- maximum visibility for your research: over $100 \mathrm{M}$ website views per year

At BMC, research is always in progress.

Learn more biomedcentral.com/submissions 